\title{
Infrapatellar fat pad area on knee MRI: does it correlate with the extent of knee osteoarthritis?
}

\author{
Eman Ragab and Dena Serag ${ }^{*}(\mathbb{D}$
}

\begin{abstract}
Background: Osteoarthritis (OA) of the knee joint is a common cause of chronic disability in older adults. During the past 10 years, the infrapatellar fat pad (IPFP) has emerged as a new player in the pathogenesis of knee OA. Its exact role in the pathogenesis of knee OA remains uncertain. While many studies focused on the detrimental effect of the chemical mediators released by IPFP and their role in the accentuation of the development of OA, only few studies elucidated the beneficial effect of IPFP maximal area as a local shock absorber protecting the adjacent articular structures from progressive damage. The aim of this study was to evaluate the relation between the IPFP maximal area and the prevalence of OA manifestations. We also studied the relation between the subcutaneous (SC) fat thicknesses on the medial aspect of the knee as a surrogate marker of body obesity and the IPFP area.

Results: A total of 216 knee scans for 188 adult patients (64 males and 124 females) who met the inclusion criteria were examined. They were between 45 and 66 years (mean 52.5 years). The mean IPFP area for all patients was 6.9 $\mathrm{cm}^{2}$ ( $\pm 1.6 \mathrm{SD}$ ) (ranged from 4.5 to $11 \mathrm{~cm}^{2}$ ). After adjustment for potential confounders, there was a significant negative association between IPFP area and radiographic manifestations of OA (osteophites, joint space narrowing, and grade of OA) ( $P$ value $<0.001$ for each), as well as MRI manifestations of OA (cartilage defects and subchondral bone marrow lesions) ( $P$ value $<0.001$ and $<0.003$, respectively). There was a negative but non-significant association between IPFP area and SC fat thickness.

Conclusion: In our study, we found supportive evidence that IPFP maximal area is associated with fewer osteoarthritic knee changes and less cartilage damage, suggesting that it plays a protective role against the development and progression of OA. Further large-scale clinical studies are awaited to confirm the obtained results. Based on our findings, it would be recommended to avoid IPFP resection during surgery in order to maintain its protective effect.
\end{abstract}

Keywords: Infrapatellar fat pad, Knee osteoarthritis, MRI

\section{Background}

Osteoarthritis (OA) is a common cause of chronic disability in older adults commonly affecting the knees [1]. It encompasses a spectrum of structural joint changes including loss of articular cartilage, formation of osteophites, reduction in joint spaces, and subchondral bone involvement [2]. Although the pathogenesis of knee OA

* Correspondence: denaserag@yahoo.com

Department of Radiodiagnosis, Faculty of Medicine, Menoufia University, Shebeen El-Kom 32511, Egypt

\section{Springer Open}

is not fully understood, both metabolic and mechanical factors play roles in the progression of this disease [1]. Age, female gender, and body weight are risk factors for knee OA [3].

Infrapatellar fat pad (IPFP), an intra-capsular but extra-synovial structure [4], is situated in the knee below the patella, lying between the patellar tendon, femoral condyles, and tibial plateau [5]. It is structurally similar to the subcutaneous fat [6]. Recent studies suggested that IPFP being located so close to the cartilaginous and

(c) The Author(s). 2021 Open Access This article is licensed under a Creative Commons Attribution 4.0 International License, which permits use, sharing, adaptation, distribution and reproduction in any medium or format, as long as you give appropriate credit to the original author(s) and the source, provide a link to the Creative Commons licence, and indicate if changes were made. The images or other third party material in this article are included in the article's Creative Commons licence, unless indicated otherwise in a credit line to the material. If material is not included in the article's Creative Commons licence and your intended use is not permitted by statutory regulation or exceeds the permitted use, you will need to obtain permission directly from the copyright holder. To view a copy of this licence, visit http://creativecommons.org/licenses/by/4.0/. 
bony surfaces may reduce the impact loading and absorb forces generated within the knee joint, thus playing a protective role against initiation and progression of $\mathrm{OA}[6,7]$.

Other studies highlight the role of the inflammatory mediators derived from IPFP and their effect on the progression of OA $[8,9]$ and considered IPFP as an active joint structure in the initiation and progression of knee OA [5], as inflammatory mediators derived from IPFP can influence the cartilage and synovium metabolism. To our knowledge, there have been few studies [7] about the association between IPFP and knee manifestations of OA, so the role of IPFP in knee OA remains largely unknown.

Structural joint abnormalities on X-ray such as joint space narrowing (JSN), marginal osteophites, and changes on MRI such as cartilage defects and subchondral bone marrow lesions (BMLs) are usually used to evaluate the presence/progression of knee OA [10].

The aim of our study was, therefore, to delineate the relation between knee joint manifestations of OA and IPFP area in older adults. We also studied the relation between $\mathrm{SC}$ fat thicknesses on the medial side of the knee joint as a surrogate marker of body obesity and IPFP area.

\section{Methods}

\section{Study population}

This retrospective observational study was conducted in the period from January 2019 to February 2020. It included 188 adult patients (64 \{34\%\} males and $124\{66 \%\}$ females), with a mean age $\{ \pm S D\}$ of 52.5 years $\{ \pm 6.6\}$ and an age range of $45-66$ years. Patients were referred to the radiology department for MRI scanning of the knee with a variety of symptoms including knee pain, clicking, and local swelling. The inclusion criterion was adult patients older than 45 years. The exclusion criteria were patients with a history of recent trauma, recent open knee surgery, or arthroscopic intervention. A total of 216 MRI scans for 188 patients who met the inclusion criterion were finally included in the study (160 patients had one knee scanned, while 28 patients underwent bilateral knee examination). The study was approved by the local Research Ethics Committee on 22 March 2020, reference number of approval 32020-RAD-3. All patients included in this study gave written informed consents to participate in this research.

\section{Anthropometric measurements}

Patients' weight was measured in kilograms, and height was measured in centimeters. The body mass index (BMI) was calculated according to the following formula: $\mathrm{BMI}=\mathrm{kg} / \mathrm{m}^{2}$, where $\mathrm{kg}$ is a patients' weight in kilograms and $\mathrm{m}^{2}$ is their height in squared meters.

\section{Radiographic knee assessment}

All patients underwent plain X-ray examination of the knee joint in the standing antero-posterior position.
Radiographs were carefully examined for the presence of osteophites and joint space narrowing (JSN). Patients were divided into three groups according to the severities of osteoarthritis (mild, moderate, and severe) using the Kellgren-Lawrence OA grading scale [11] as shown in Fig. 1 and Table 1. Both entities (osteophites and JSN) were further scored from 0 to $3(0=$ normal, while $3=$ most severe) according to the Osteoarthritis Research Society International (OARSI) Atlas [12].

\section{Magnetic resonance imaging assessment Examination protocol}

MRI scans were performed using a 1.5-T unit (TOSHIBA, Excelart Vantage). Patients were examined in the supine position feet first, using a dedicated extremity coil. Before MR examination, patients were asked to remove any metallic objects around the scan area. None of the patients received intravenous contrast.

\section{The standard MRI protocol of the knee}

The following are the standard MRI protocol of the knee: sagittal T1WI (TR $=605 \mathrm{~m} \mathrm{~s}, \mathrm{TE}=14 \mathrm{~m} \mathrm{~s}, \mathrm{FOV}=$ $18 \mathrm{~cm}$, slice $\mathrm{T}=3 \mathrm{~mm}$, matrix $=230 \times 256$ pixels), sagittal T2WI $(\mathrm{TR}=2900 \mathrm{~m} \mathrm{~s}, \mathrm{TE}=31 \mathrm{~m} \mathrm{~s}, \mathrm{FOV}=18 \mathrm{~cm}$, slice $\mathrm{T}=3 \mathrm{~mm}$, matrix $=384 \times 512$ pixels), sagittal T2WI with fat saturation $(\mathrm{TR}=3600 \mathrm{~m} \mathrm{~s}, \mathrm{TE}=31 \mathrm{~m} \mathrm{~s}, \mathrm{FOV}=20 \mathrm{~cm}$, slice $\mathrm{T}=3 \mathrm{~mm}$, matrix $=384 \times 512$ pixels), sagittal $\mathrm{PD}$ $(\mathrm{TR}=8100 \mathrm{~m} \mathrm{~s}, \mathrm{TE}=102 \mathrm{~m} \mathrm{~s}, \mathrm{FOV}=20 \mathrm{~cm}$, slice $\mathrm{T}=3$ $\mathrm{mm}$, matrix $=404 \times 512$ pixels $)$, axial T2WI $(\mathrm{TR}=2900$ $\mathrm{m}$ s, $\mathrm{TE}=100 \mathrm{~m}$ s, $F O V=20 \mathrm{~cm}$, slice $\mathrm{T}=3.5 \mathrm{~mm}$, matrix $=520 \times 640$ pixels $)$, and coronal STIR $(\mathrm{TR}=3700$ $\mathrm{m} \mathrm{s}, \mathrm{TE}=41 \mathrm{~m} \mathrm{~s}, \mathrm{TI}=160 \mathrm{~m} \mathrm{~s}, \mathrm{FOV}=20 \mathrm{~cm}$, slice $\mathrm{T}=4$ $\mathrm{mm}$, matrix $=410 \times 512$ pixels).

Images were transferred by a computer network to the workstation (Aze Virtual Place FujinRaijin 310) on which images were carefully reviewed. A trained radiologist (with more than 15 years of experience in musculoskeletal radiology) examined the scans. IPFP area was measured on fat-saturated mid-sagittal T2WI FSE by drawing a manual trace around the borders of IPFP as shown in Fig. 2.

Cartilage defects were assessed in the medial and lateral compartments of the knee joint as well as the patellofemoral articulation using axial and sagittal T2weighted fast spin-echo images. They were graded on a 0 to 4 scale as follows: grade $0=$ normal cartilage, grade $1=$ focal blistering and intra-cartilaginous high-signal area with an intact surface, grade $2=$ irregularities on the surface or bottom and loss of $<50 \%$ of thickness; grade $3=$ deep ulceration with loss of $>50 \%$ of thickness, and grade 4 = full-thickness chondral defect with denuding of subchondral bone.

Abnormal subchondral bone marrow lesions (BMLs) were defined as areas of increased signal intensity in the 


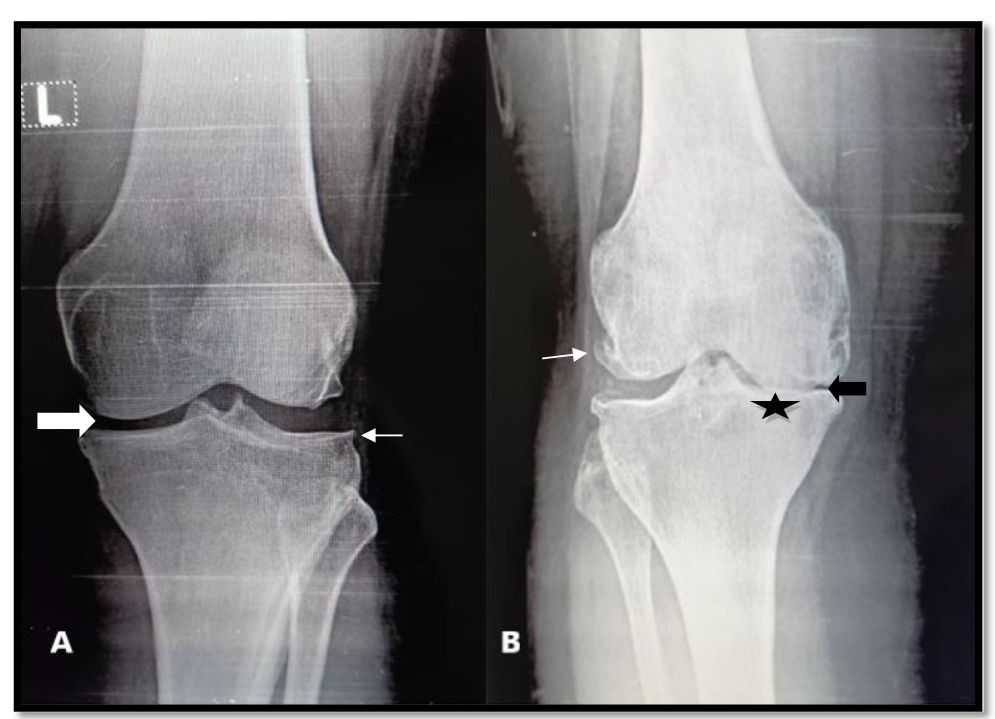

Fig. 1 The Kellgren- Lawrence radiographic osteoarthritis grading scale. a Mild osteoarthritis with small osteophites (arrows) and possible medial joint space narrowing (bold arrow). b Severe osteoarthritis with large osteophites (white arrow), marked joint space narrowing (black arrow), and subchondral sclerosis (astride)

subarticular regions (in the tibial and femoral condyles and posterior patellar surface). They were assessed in coronal STIR sequence and given a score from 0 to 3 $(0=$ normal and $3=$ most severe $)$.

Figures 3 and 4 show mild and advanced OA on MRI with the corresponding IPFP areas.

SC thickness was measured at the medial aspect of the knee joint and used as a surrogate of body obesity. It was measured on axial T2WI starting from the skin to the bony cortex of the postero-medial aspect of the medial femoral condyle as shown in Fig. 5.

\section{Statistical analysis}

The results were analyzed with SPSS statistical package version 23 (SPSS Inc. Released 2015; IBM SPSS statistics for Windows, version 23.0, Armonk, NY: IBM Corp).

Data was expressed in number (No.), percentage (\%), mean $(x)$, and standard deviation (SD). Student's $t$ test and Mann-Whitney's test were used for comparison of quantitative variables between two groups of normally and not normally distributed data, respectively. The ANOVA test was used for comparison of quantitative variables between more than two groups of normally distributed data with the Tukey test as a post hoc test while the Kruskal-Wallis test was used for comparison of quantitative variables between more than two groups of not normally distributed data with Tamhane's test as a post hoc test. Pearson correlation was used to show the correlation between two continuous normally distributed variables while Spearman correlation was used for not normally distributed ones. Univariate and multivariate linear regression models were performed to ascertain the effects of possible determinants on the IPFP area. A two-sided $P$ value of $<0.05$ was considered statistically significant.

\section{Results}

This study included 216 knee examinations for 188 adult patients (64 \{34\%\} males and $124\{66 \%\}$ females), with a mean age $\{ \pm S D\}$ of 52.5 years $\{ \pm 6.6\}$. Their ages ranged from 45 to 66 years. Patients' demographics and characteristics are summarized in Table 2.

The mean IPFP area for all patients was $6.9 \mathrm{~cm}^{2}(+1.6$ $\mathrm{SD}$ ), with a range from 4.5 to $11 \mathrm{~cm}^{2}$ (the mean IPFP areas for mild, moderate, and severe OA were $7.9 \mathrm{~cm}^{2}$, $5.9 \mathrm{~cm}^{2}$, and $5.3 \mathrm{~cm}^{2}$, respectively). There was a negative correlation between the IPFP area and the age of the patients $(P$ value $<0.001)(r=-0.401)$.

BMI of the patients ranged from 22 to 33 with a mean of $27( \pm 2.68 \mathrm{SD})$. BMI was significantly associated with

Table 1 The Kellgren-Lawrence radiographic osteoarthritis grading scale

\begin{tabular}{ll}
\hline Kellgren-Lawrence osteoarthritis grading scale & Description \\
\hline Mild OA & -Definite osteophites and possible narrowing of the joint space \\
Moderate OA & -Moderate multiple osteophites, definite joint space narrowing, and subchondral sclerosis \\
Severe OA & -Large osteophites, marked joint space narrowing, severe sclerosis, and bone deformity \\
\hline
\end{tabular}




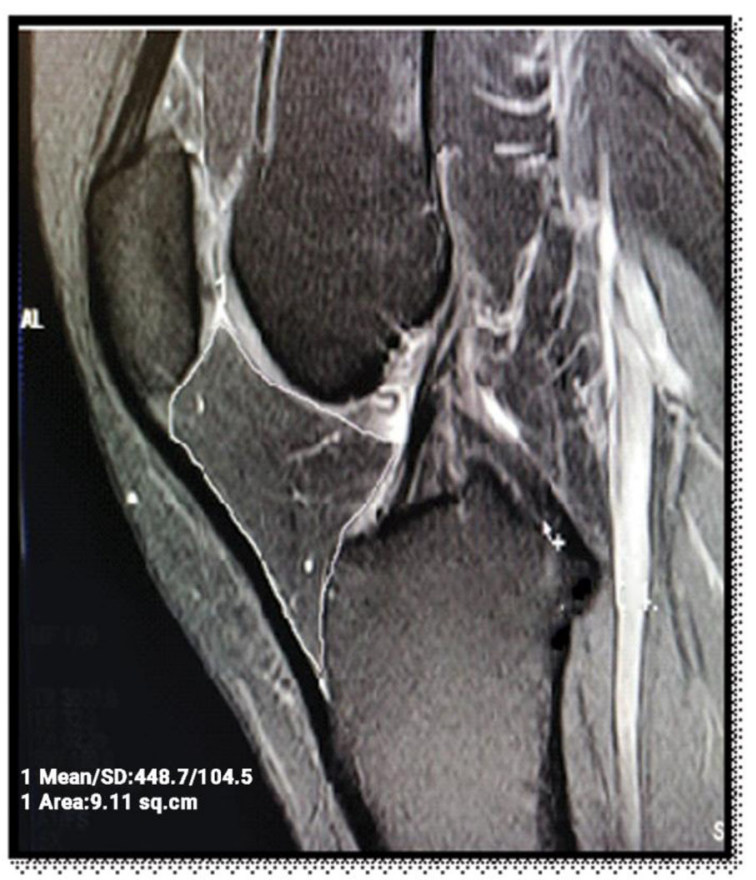

Fig. 2 Measurement of the infrapatellar fat pad area on MRI. a Sagittal T2WI with fat saturation showing manual tracing of the boundaries of the IPFP (the maximum area represents the infrapatellar fat pad area in square centimeter)

gender $(P$ value $<0.001)$. There was a significant association between the BMI and the IPFP area $(P$ value $<$ 0.001). Higher BMI negatively correlated with the IPFP areas $(P$ value $=0.018)(r=-0.161)($ Tables 3 and 4$)$.

Post hoc analysis revealed that the mean IPFP area varied significantly among patients with different grades of BMI. Patients with normal BMI had a significantly larger IPPF area than overweight or obese patients $(P=$ 0.005 and $<0.001$ respectively). Also, overweight patients had a significantly larger mean IPPF area than obese patients $(P=0.01)$ (Table 3$)$.

There was no significant association between the gender and the IPFP area $(P$ value $=0.2)($ Table 3$)$.

Table 3 also describes the relation between the radiographic OA grade, JSN, osteophites, and IPFP area. In univariate analyses, the IPFP area was significantly and negatively associated with the grade of radiographic OA ( $P$ value $<0.001)$, JSN ( $P$ value $<0.001)$, and the presence of osteophites $(P$ value $<0.001)$. Larger IPFP areas were significantly associated with reduced grades of OA, lesser degree of JSN, and less osteophite formation. In multivariate analyses (after adjustment for the potential confounders), the negative correlation between IPFP area and OA grade remained significant $(P$ value $<0.001)$. However, the correlation between the IPFP area and the presence of osteophites and JSN was consistent but did not reach significance (Table 5).
Post hoc analysis revealed that increasing grades of $\mathrm{OA}$ was associated with a significant reduction in the IPPF area. Patients with a mild degree had a significantly larger IPPF area than patients with moderate or severe grades $(P<0.001$ for both); also, patients with moderate grade had a larger IPPF area than patients with severe grade $(P=0.002)$ (Table 3$)$.

Table 4 describes the correlation between the IPFP area and patients' age, BMI, and individual manifestations of OA.

IPFP area was negatively and significantly associated with the presence of cartilage defects $(r=-0.515, \mathrm{CI}=$ 95\%) ( $P$ value $<0.001)$ (Table 4$)$. In the univariate analysis, larger IPFP areas correlated with fewer cartilage defects in medial and lateral joint spaces as well as the patello-femoral articulation. In the multivariate analysis (after adjustment for radiographic OA), the correlation between the IPFP area and the presence of cartilage defects was consistent but did not reach significance $(P=$ 0.184) (Table 5).

There was a significant negative association between the IPFP area and the presence of subchondral BMLs $(r=-0.380, \mathrm{CI}=95 \%)(P$ value $<0.003)($ Table 4$)$. In the univariate analysis, larger IPFP areas correlated with less BMLs. In the multivariate analysis (after adjustment for radiographic OA), the correlation between the IPFP area and the presence of BMLs was consistent but did not reach significance $(P=0.89)$ (Table 5). 


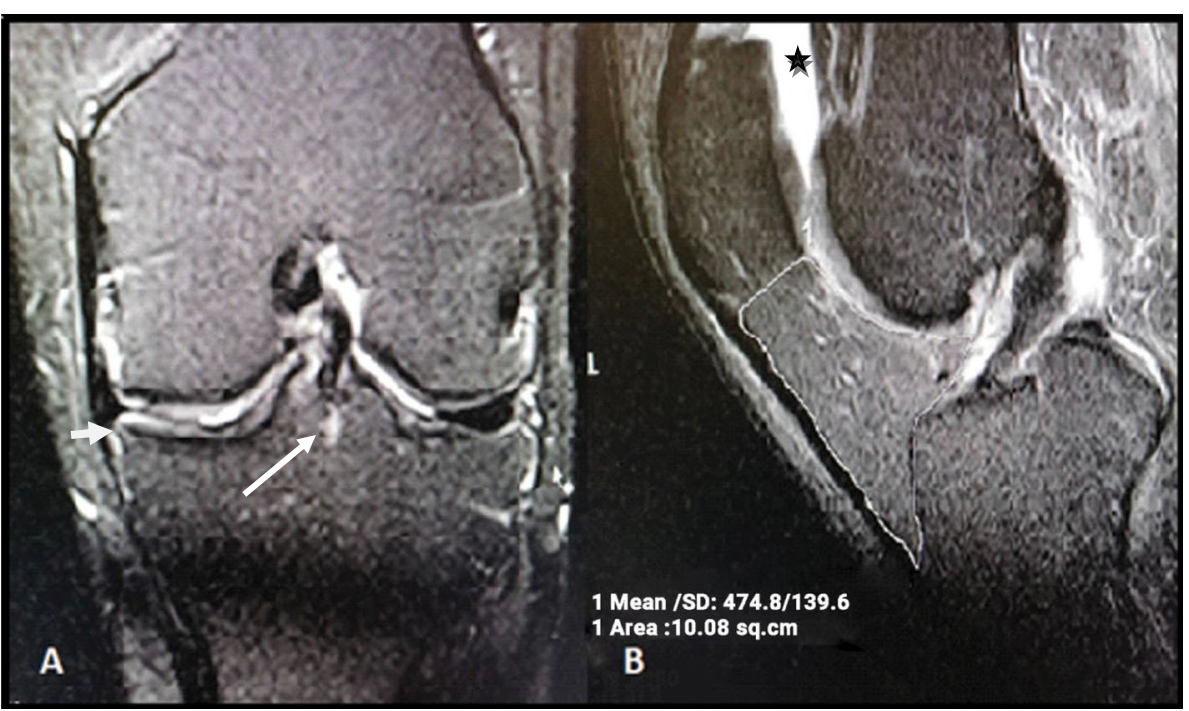

Fig. 3 Mild osteoarthritis on MRI. a Coronal STIR image showing early OA with small marginal osteophite (short arrow) and small subchondral BML (long arrow). b Sagittal T2WI with fat saturation showing the corresponding IPFP area $=10 \mathrm{~cm}^{2}$ (astride $=$ knee effusion)

Regarding BMLs and cartilage defects, post hoc analysis showed that increasing severity grades of either factor was associated with a significant reduction of IPFP area $(P<0.001$ and 0.003 respectively). Patients without BMLs had significantly larger IPPF areas than grade 1,2 , or 3 ( $P<0.001$ for each), patients with grade 1 had significantly larger IPPF areas than grade 2 or $3(P<0.001$ for both), and finally, grade 2 had significantly larger IPFP areas than grade $3(P<0.001)$. Regarding cartilage defects, patients without cartilage defects (grade 0 ) or with grade 1 defect had significantly larger IPPF areas than grade 2,3 , or 4 ( $P<0.001$ for each), and finally, patients with grade 2 had significantly larger IPPF areas than patients with grade $4(P<0.001)$ (Table 3$)$.

The SC thickness on the medial side of the knee joint was measured in the axial plane and used as a surrogate of body obesity.

There was a negative correlation between the SC fat thickness and the IPFP area. In the univariate analysis, smaller IPFP areas significantly and negatively correlated with $\mathrm{SC}$ fat thickness $(P$ value $<0.003, \mathrm{CI}=$ 95\%) $(r=-0.201)$ (Table 4). In the multivariate analysis (after adjustment for the patients' age and gender), the negative correlation between the IPFP area and the SC fat thickness was consistent but did not reach significance $(P=0.14)$.

\section{Discussion}

The role of IPFP in the pathogenesis of knee OA remains uncertain. Many authors suggest that IPFP maximal area appears to play a protective role against the development and progression of knee OA and cartilage damage owing to its intra-capsular location being close to the femoral condyles and tibial plateau [13]. Others assume that IPFP enlargement may result in metabolic changes characterized by chronic, low-level inflammation due to increased local levels of adipose-derived cytokines, or "adipokines," making those patients more prone to symptomatic OA [7]. This study aimed to delineate the relation between the IPFP area and the radiological manifestations of knee OA. We also studied the relation between the SC fat thickness, as a surrogate of body obesity, and IPFP area.

In the current study, we retrospectively reviewed 216 knee examinations for 188 adult patients (64 \{34\%\} males and $124\{66 \%\}$ females), with a mean age $\{+$ SD $\}$ of 52.5 years $\{ \pm 6.6 \mathrm{SD}\}$ (ranging from 45 to 66 years). The mean IPFP area for all patients was $6.9 \mathrm{~cm}^{2}\{ \pm 1.6 \mathrm{SD}\}$, with a range from 4.5 to $11 \mathrm{~cm}^{2}$. This was consistent with the study results obtained by Han et al. They analyzed 977 knee MRI scans with a mean IPFP area $=7.59$ $\mathrm{cm}^{2}$ (SD 1.18, range 4.56 to 12.14) [14].

There was a negative correlation between the IPFP area and the age of the patients $(P$ value $<0.001$, $r=-0.401)$. On the contrary to our findings, Han et al. reported a significant positive association between IPFP volume and age $(P<0.05, \mathrm{CI} 95 \%, r 0.35)[14]$.

In our study, there was a significant association between the BMI and the IPFP area $(P$ value $<0.001)$. Higher BMI negatively correlated with the IPFP areas $(r=-0.161, P$ value $=0.018)$. Similarly, Masaki et al. reported statistically significant negative correlation between IPFP volume and BMI $(r=-0.29 ; P<0.001)$ [15]. While other results reported by Han et al. found no significant association between IPFP area and BMI 


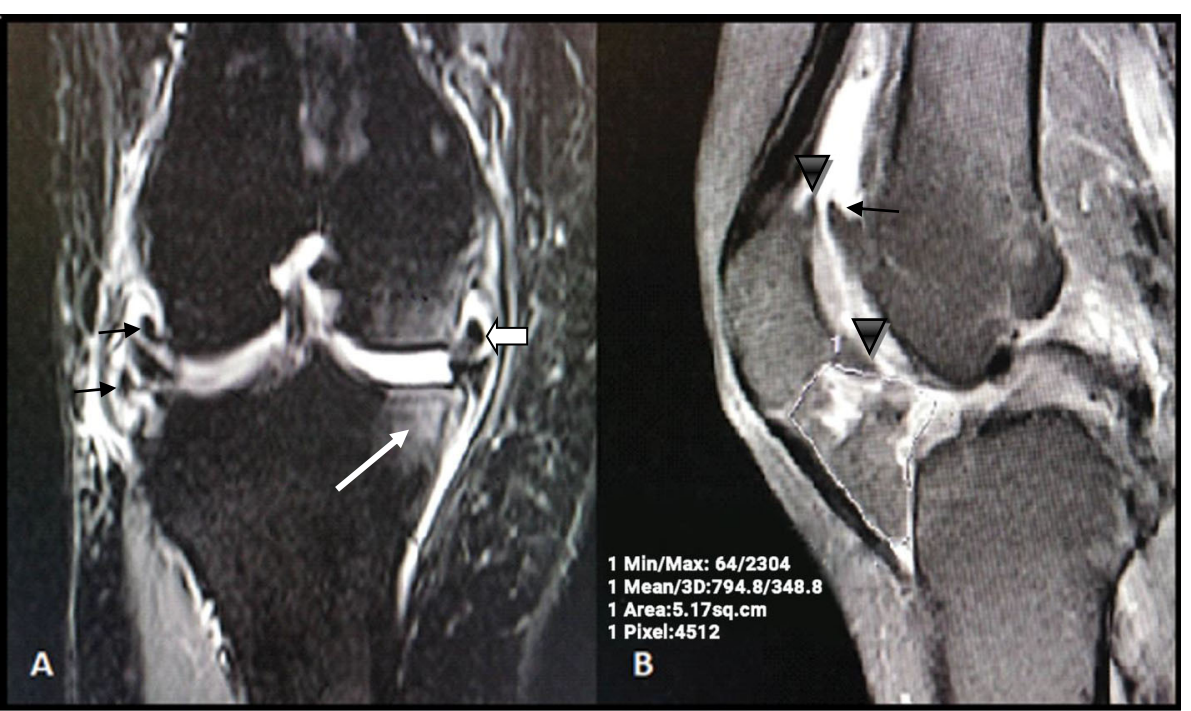

Fig. 4 Advanced osteoarthritis on MRI. a Sagittal STIR image showing advanced osteoarthritic changes with bulky marginal osteophites (short arrows), subchondral BML (long arrow), and intra-articular loose body (bold arrow). b Sagittal T2WI with fat saturation showing patellar polar spurs (black arrowheads), corresponding IPFP area $=5.17 \mathrm{~cm}^{2}$

[14]. Recent studies from the USA and the Netherlands also reported no association between IPFP volume and BMI [7].

There was no significant association between the gender and the IPFP area $(P$ value $=0.2)$. This was quite different from the results reported by Duran et al. [16]. They reported a significant difference in the mean IPFP area between males and females $(17.8 \pm 4.5 \mathrm{SD}$ for males vs $13.4 \pm 3.7 \mathrm{SD}$ for females) ( $P$ value $<0.05)$. Han et al. reported similar findings. They stated that increased IPFP area was negatively associated with the female gender [14]. The difference in results may be attributed to the smaller sample size in our study.

In our study, we found consistent evidence that IPFP maximal area is beneficially associated with reduced radiological manifestations of knee OA. It appears to play a protective role against the development of $\mathrm{OA}$ and cartilage breakdown as it mechanically functions as a local shock absorber by reducing the axial forces on the knee joint.

In our study, there was a significant negative correlation between radiographic manifestations of OA (osteophites, JSN, and grade of OA) and IPFP area ( $P$ value $<0.001)$. In the multivariate analyses, the negative correlation between IPFP area and OA grade remained significant $(P$ value $<0.001)$, yet the correlation between the IPFP area and the presence of osteophites and JSN was consistent but did not reach significance. Our results were in agreement with the results obtained by Han et al. [14]. They studied 977 patients with different grades of knee OA and reported that maximal IPFP area was significantly and negatively associated with the prevalence of radiographic OA, presence of osteophites, and JSN in both the medial and lateral tibiofemoral compartments providing evidence in support of the protective effect of local joint fat on knee joint structures [14].

Cai et al. [13] also reported a significant negative correlation between the IPFP area and the presence of osteophites at the medial and lateral tibio-femoral compartments $(P<0.05)$, but they found no significant association between IPFP area and JSN.

In contrast to our results, Chuckpaiwong et al. [7] reported that there was no significant difference in IPFP volume between $\mathrm{OA}$ patients and the control group. The difference may be attributed to the small sample size in their study (15 patients and 15 controls).

The current study revealed that IPFP area was negatively and significantly associated with the presence of cartilage defects $(P$ value $<0.001)$. Our results came in agreement with the results recorded by Pan et al. [17]. They reported a significant association between IPFP area and reduced risks of development of medial joint space cartilage defects (relative risk 0.59, $P<0.05$ ).

Han et al. [14] found that a larger IPFP area was significantly associated with decreased medial and lateral tibial plateau cartilage defects after adjustment for radiographic OA $(P<0.01)$ which was in concordance with our results. This further supports that IPFP is protective against knee structural changes in OA.

We found that subchondral BMLs were significantly and negatively associated with IPFP area $(r=-0.380$, $\mathrm{CI}=95 \%)(P$ value $<0.003)$. This was consistent with the 


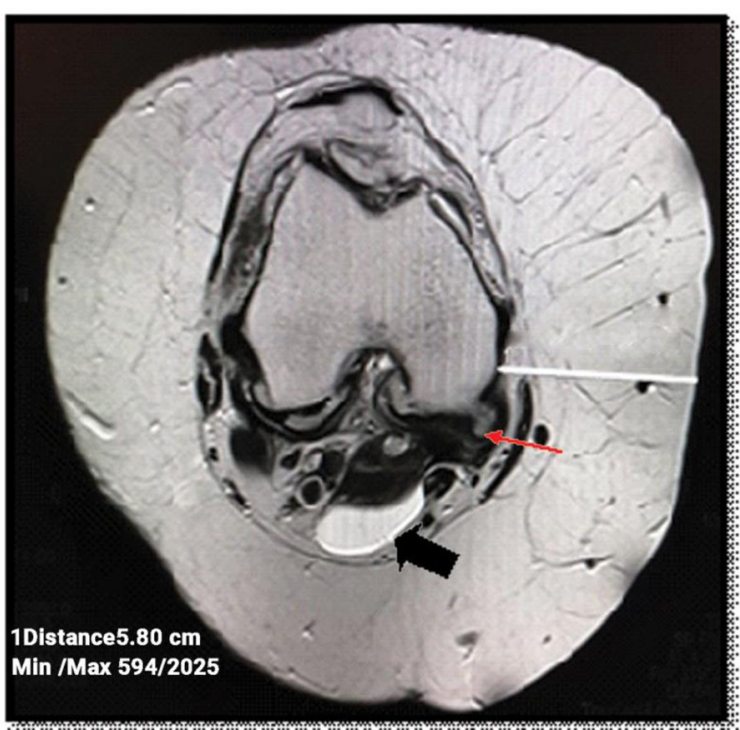

Fig. 5 Measurement of the subcutaneous fat thickness. Axial T2WI of the knee showing subcutaneous fat measurement on the medial aspect of the knee joint behind the medial femoral condyle. Osteophite (red arrow), backer cyst (bold arrow)

results reported by Han et al. [14]. They found that there was a significant negative association between IPFP area and total BML scores for all joint compartments after adjustment for co-variants.

In the present study, there was a weak negative correlation between the SC fat thickness and the IPFP area $(P$ value $<0.01, \mathrm{CI}=95 \%)(r=-0.201)$. However, in the multivarite analysis, the negative correlation was consistent but did not reach significance $(P=0.14)$. Few studies reported

Table 2 Patients' characteristics for the 216 scans included in the study

\begin{tabular}{ll}
\hline Characteristic & Value \\
\hline Age $^{a}$ & 52.5 years $( \pm 6.6$ SD) \\
Gender, $n(\%)$ & \\
$\quad$ Male & $64(34 \%)$ \\
$\quad$ Female & $124(66 \%)$ \\
BMI mean ( \pm SD, range) & $27( \pm 2.68$ SD, 22.0-33.0) \\
Joint space narrowing, $n(\%)$ & $148(68.5 \%)$ \\
Osteophites, $n(\%)$ & $208(96 \%)$ \\
Cartilage defects, $n(\%)$ & $132(61 \%)$ \\
Bone marrow lesions, $n(\%)$ & $184(85 \%)$ \\
Osteoarthritis grade & \\
$\quad$ Mild & $116(53.7 \%)$ \\
Moderate & $76(35.3 \%)$ \\
$\quad$ Severe & $24(11 \%)$ \\
\hline
\end{tabular}

${ }^{\mathrm{a}}($ Mean $\pm \mathrm{SD})$ and ( $n$ and \%) number and percentage
Table 3 Association between IPFP area and different manifestations of osteoarthritis, gender, and BMl

\begin{tabular}{|c|c|c|}
\hline Variables & IPFP $($ mean \pm SD) & $P$ value \\
\hline \multicolumn{3}{|l|}{ BMI category } \\
\hline Normal (18.5-25) & $7.66 \pm 1.35$ & \multirow[t]{3}{*}{$<0.001$} \\
\hline Overweight (25-30) & $6.90 \pm 1.63$ & \\
\hline Obese $(>30)$ & $6.15 \pm 1.31$ & \\
\hline \multicolumn{3}{|l|}{ Gender } \\
\hline Male & $7.24 \pm 1.73$ & \multirow[t]{2}{*}{0.202} \\
\hline Female & $6.92 \pm 1.56$ & \\
\hline \multicolumn{3}{|l|}{ Osteophites } \\
\hline 0 & $7.43 \pm 0.13$ & \multirow[t]{4}{*}{$<0.001$} \\
\hline 1 & $7.80 \pm 1.59$ & \\
\hline 2 & $5.84 \pm 0.58$ & \\
\hline 3 & $5.70 \pm 0.83$ & \\
\hline \multicolumn{3}{|l|}{ Joint space narrowing } \\
\hline 0 & $8.03 \pm 1.52$ & \multirow[t]{4}{*}{$<0.001$} \\
\hline 1 & $6.76 \pm 1.39$ & \\
\hline 2 & $5.57 \pm 0.55$ & \\
\hline 3 & $5.40 \pm 0.81$ & \\
\hline \multicolumn{3}{|l|}{ OA grade } \\
\hline Mild & $7.93 \pm 1.47$ & \multirow[t]{3}{*}{$<0.001$} \\
\hline Moderate & $5.91 \pm 0.63$ & \\
\hline Severe & $5.33 \pm 0.65$ & \\
\hline \multicolumn{3}{|l|}{ Bone marrow lesions } \\
\hline 0 & $7.63 \pm 1.67$ & \multirow[t]{4}{*}{$<0.001$} \\
\hline 1 & $7.27 \pm 1.68$ & \\
\hline 2 & $6.14 \pm 0.81$ & \\
\hline 3 & $5.15 \pm 0.1$ & \\
\hline \multicolumn{3}{|l|}{ Cartilage defects } \\
\hline 0 & $7.61 \pm 1.48$ & \multirow[t]{5}{*}{0.003} \\
\hline 1 & $7.63 \pm 1.93$ & \\
\hline 2 & $6.02 \pm 0.85$ & \\
\hline 3 & $5.82 \pm 0.72$ & \\
\hline 4 & $5.50 \pm 0.01$ & \\
\hline
\end{tabular}

on the relation between IPFP area and SC fat thickness. Our results came in agreement with the study carried out by Teichtahl et al. [18]. They stated that the lack of significant association between measures of body adiposity (SC fat, trunk fat) and the size of the IPFP might suggest that the IPFP size is not simply a marker of systemic obesity. Similarly, Masaki et al. [15] stated that IPFP is maintained even in starved conditions where SC fat diminishes.

Han et al. [14] also reported similar findings. They found that IPFP area was not associated with systematic fat mass. They also stated that systemic metabolic changes do not necessarily affect the size of IPFP. 
Table 4 Correlation between the IPFP area and both patient and disease characteristics

\begin{tabular}{lll}
\hline Variables & IPFP area & \\
\cline { 2 - 3 } & $\boldsymbol{r}$ & $\boldsymbol{P}$ value \\
\hline Patients' characteristics & -0.401 & $<0.001$ \\
Age & -0.161 & 0.018 \\
BMI & & \\
X-ray & -0.587 & $<0.001$ \\
Joint space narrowing & -0.668 & $<0.001$ \\
Osteophites & -0.695 & $<0.001$ \\
OA grade & & \\
MRI & -0.380 & $<0.001$ \\
BM lesions & -0.515 & $<0.001$ \\
Cartilage defect & -0.201 & 0.003 \\
SC fat thickness &
\end{tabular}

$r$ correlation co-efficient, $O A$ osteoarthritis, $B M$ bone marrow, SC subcutanous

The protective effect of the maximal IPFP area on the knee joint structures can be explained by its intracapsular location and close proximity to the articular bony surfaces. Its flexible and displaceable nature helps to act as a local shock absorber minimizing the mechanical stress over the adjacent knee joint structures. It is also suggested that chemical mediators conditioned by IPFP inhibit catabolic processes in cartilage [19].

Based on our results, we concluded that the maximal IPFP area plays a protective rather than harmful role against the initiation or progression of OA. During open and arthroscopic knee surgery, the IPFP is usually partially or totally resected to provide a clear visualization of internal joint structures [20]. It would be recommended to spare IPFP during surgery to maintain its protective effect.

\section{Limitations}

Our study was limited by the lack of software used for 3dimensional measurement of IPFP volume. Instead, we depended on measuring the IPFP maximal area on two dimensional mid-sagittal T2WI MRI by manually tracing the boundaries of the IPFP. We also depended only on measuring the IPFP area and did not include other imaging features about the quality of IPFP like edema, fibrosis, and signal intensity changes that might be influenced by OA.

\section{Conclusion}

In our study, we found supportive evidence that IPFP maximal area is associated with fewer osteoarthritic knee changes and less cartilage damage, suggesting that it plays a protective role against the development and progression of OA. Further large-scale clinical studies are awaited to confirm the obtained results.

\section{Recommendations}

Based on our findings, it would be recommended to avoid IPFP resection during surgery in order to maintain its protective role. Regarding the association between the IPFP area and the presence and development of knee $\mathrm{OA}$, we can conclude the value of measuring the IPFP area in early detection of knee OA in asymptomatic patients, which helps early management and prevention of OA disease progression, especially in patients vulnerable to early OA changes as in athletes and those with a family history of early OA. We also advise to perform the chemical synovectomy (by intra-articular injection of methotrexate) instead of surgical synovectomy in patients with chronic arthritis to preserve the IPFP.

Table 5 Univariate vs multivariate regression model of the IPFP area and patient demographics as well as individual manifestations of $\mathrm{OA}$

\begin{tabular}{|c|c|c|c|c|c|c|}
\hline \multirow[t]{2}{*}{ Variables } & \multicolumn{3}{|l|}{ Univariate } & \multicolumn{3}{|l|}{ Multivariate } \\
\hline & Unstandardized B & Standardized B & $P$ value & Unstandardized B & Standardized B & $P$ value \\
\hline Age & -0.088 & -0.367 & $<0.001$ & -0.016 & -0.062 & 0.396 \\
\hline Gender & -0.320 & -0.093 & 0.202 & -0.279 & -0.081 & 0.271 \\
\hline $\mathrm{BMI}$ & -0.096 & -0.161 & 0.018 & 0.161 & 0.259 & 0.167 \\
\hline \multicolumn{7}{|l|}{ X-ray } \\
\hline Joint space narrowing & -1.048 & -0.536 & $<0.001$ & -0.058 & -0.25 & 0.935 \\
\hline Osteophites & -1.089 & -0.552 & $<0.001$ & 0.653 & 0.309 & 0.416 \\
\hline OA Grading & -1.533 & -0.658 & $<0.001$ & -2.241 & -0.848 & $<0.001$ \\
\hline \multicolumn{7}{|l|}{ MRI } \\
\hline Cartilage defects: & -0.666 & -0.477 & $<0.001$ & -0.177 & -0.127 & 0.184 \\
\hline BM lesions & -0.858 & -0.394 & 0.003 & -0.022 & -0.010 & 0.895 \\
\hline SC fat thickness & -0.150 & -0.160 & 0.019 & -0.286 & -0.284 & 0.142 \\
\hline
\end{tabular}




\section{Abbreviations}

MRI: Magnetic resonance imaging; IPFP: Infrapatellar fat pad;

OA: Osteoarthritis; BMLs: Bone marrow lesions; JSN: Joint space narrowing; BMI: Body mass index; FOV: Field of view; TI: Time of inversion; STIR: Shorttime inversion recovery; SC: Subcutanous

\section{Acknowledgements}

The authors would like to thank Dr. Dalia Salah (MD in Rheumatology) for illustrating the applications of the IPFP area in clinical practice.

\section{Authors' contributions}

ER and DS contributed equally to the study design, data collection, analysis, and interpretation of the results. All authors read and approved the final manuscript.

\section{Funding}

There is no funding

\section{Availability of data and materials}

Data will be available upon request via contacting the corresponding author.

\section{Ethics approval and consent to participate}

This study was approved by the Research Ethics Committee of the Faculty of Medicine at Menoufia University in Egypt on 22 March 2020, reference number of approval 32020-RAD-3. All patients included in this study gave written informed consent to participate in this research.

\section{Consent for publication}

All patients included in this research gave written informed consent to publish the data contained within this study.

\section{Competing interests}

The authors declare that they have no competing interests.

Received: 8 August 2020 Accepted: 6 December 2020

Published online: 05 January 2021

References

1. Loeser R, Hunter D (2011) Aging and osteoarthritis. Curr Opin Rheumatol 23 492-496

2. Srikanth V, Fryer J, Zhai G, Winzenberg T, Hosmer D, Jones G (2005) A metaanalysis of sex differences prevalence, incidence and severity of osteoarthritis. Osteoarthr Cartil 13:769-781

3. Ding C, Cicuttini F, Scott F, Cooley H, Jones G (2005) Knee structura alteration and BMI: a cross-sectional study. Obes Res 13:350-361

4. Saddik D, McNally E, Richardson M (2004) MRI of Hoffa's fat pad. Skeletal Radiol 33:433-444

5. Clockaerts S, Bastiaansen Y, Runhaar J, Van Osch G, Van Offel J, Verhaar J, De Clerck L, Somville J (2010) The infrapatellar fat pad should be considered as an active osteoarthritic joint tissue: a narrative review. Osteoarthr Cartil 18:876-882

6. Vahlensieck M, Linneborn G, Schild H, Schmidt H (2002) Hoffa's recess: incidence, morphology and differential diagnosis of the globular-shaped cleft in the infrapatellar fat pad of the knee on MRI and cadaver dissections. Eur Radiol 12:90-93

7. Chuckpaiwong B, Charles H, Kraus V, Guilak F, Nunley J (2010) Ageassociated increases in the size of the infrapatellar fat pad in knee osteoarthritis as measured by 3 T MRI. J Orthop Res 28:1149-1154

8. Distel E, Cadoudal T, Durant S, Poignard A, Chevalier X, Benelli C (2009) The infrapatellar fat pad in knee osteoarthritis: an important source of interleukin-6 and its soluble receptor. Arthritis Rheum 60:3374-3377

9. Gandhi R, Takahashi M, Virtanen C, Syed K, Davey J, Mohamed N (2011) Microarray analysis of the infrapatellar fat pad in knee osteoarthritis: relationship with joint inflammation. J Rheumatol 38:1966-1972

10. Ding C, Zhang Y, Hunter D (2013) Use of imaging techniques to predict progression in osteoarthritis. Curr Opin Rheumatol 25:127-135

11. Kohn M, Sassoon A, Fernando N (2016) Classifications in brief: Kellgren-Lawrence classification of osteoarthritis. Clin Orthop Relat Res 474(8):1886-1893

12. Altman R, Hochberg M, Murphy W, Wolfe F, Lequesne M (1995) Atlas of individual radiographic features in osteoarthritis. Osteoarthr Cartil 3:3-70

13. Cai J, Xu J, Wang K, Zheng S, He F, Huan S, Xu S, Zhang H, Laslett L, Ding C (2015) Association between infrapatellar fat pad volume and knee structural changes in patients with knee osteoarthritis. J Rheumatol 42(10):1878-1884

14. Han W, Cai S, Liu Z, Jin X, Wang X, Antony B, Cao Y, Aitken D, Cicuttini F, Jones G, Ding C (2014) Infrapatellar fat pad in the knee: is local fat good or bad for knee osteoarthritis? Arthritis Res Ther 16:145-148

15. Masaki T, Takahashi K, Hashimoto S, Ikuta F, Watanabe A, Kiuchi S, Okuaki T, Mochizuki Y, Takai S (2018) Volume change in infrapatellar fat pad is associated not with obesity but with cartilage degeneration. J Orthop Res 37(3):593-600

16. Duran S, Aksahin E, Kocadal O, Aktekin C, Hapa O, Gencturk Z (2015) Effect of body mass index, infra-patellar fat pad volume and age on patellar cartilage defects. Acta Orthop Belg 81:41-46

17. Pan F, Han W, Wang X, Liu Z, Jin X, Antony B, Cicuttini F, Jones G, Ding C (2015) A longitudinal study of the association between infrapatellar fat pad maximal area and changes in knee symptoms and structure in older adults. Ann Rheum Dis 74(10):1818-1824

18. Teichtahl A, Wulidasari E, Brady S, Cicuttinin F, Wang Y, Wluka A, Ding C, Giles $G$ (2015) A large infrapatellar fat pad protects against knee pain and lateral tibial cartilage volume loss. Osteoarthr Cartil 23(1):283-287

19. Bastiaansen-Jenniskens Y, Clockaerts S, Feijt C, Zuurmond A, StojanovicSusulic V, Bridts C, De-Clerck L, De-Groot J, Verhaar J, Kloppenburg M, VanOsch G (2012) Infrapatellar fat pad of patients with end-stage osteoarthritis inhibits catabolic mediators in cartilage. Ann Rheum Dis 71:288-294

20. Binks M, Vlok R, Holyoak R, Melhuish T, White L (2017) Pre-operative MRI to plan infrapatellar fat pad resection during total knee arthroplasty. Ann Rheum Dis 76:29-34

\section{Publisher's Note}

Springer Nature remains neutral with regard to jurisdictional claims in published maps and institutional affiliations.

\section{Submit your manuscript to a SpringerOpen ${ }^{\circ}$ journal and benefit from:}

- Convenient online submission

- Rigorous peer review

- Open access: articles freely available online

- High visibility within the field

- Retaining the copyright to your article

Submit your next manuscript at $\boldsymbol{\nabla}$ springeropen.com 